\title{
Chiral Effective Field Theory after Thirty Years: Nuclear Lattice Simulations
}

\author{
Dean Lee
}

Received: date / Accepted: date

\begin{abstract}
The introduction of chiral effective field theory by Steven Weinberg three decades ago has had a profound and lasting impact on nuclear physics. This brief review explores the impact of Weinberg's work on the field of nuclear lattice simulations. Rather than a summary of technical details, an effort is made to present the conceptual advances that made much of the recent progress possible.
\end{abstract}

Keywords chiral effective field theory · lattice simulations · nuclear structure $\cdot$ nuclear scattering and reactions $\cdot$ nuclear thermodynamics

\section{Introduction}

The author first learned of Weinberg's chiral effective field theory [ 81, 82, 83 from the Loeb lectures that Weinberg delivered at Harvard in 1993. While the author was a first-year graduate student at the time and didn't follow all the details, he appreciated the bold conclusion that there was an underlying hierarchy of nuclear forces. Weinberg was showing which of the many possible nuclear forces were most important at low energies. There was, however, some uncertainty if Weinberg's proposed hierarchy could withstand the combinatorial enhancement of higher-body forces in larger nuclei and many-body systems. While the field has made great strides since then, this fundamental question still remains unresolved and is an active area of research today. This article is a brief review dedicated to the memory of the inspirational physicist and pioneer we lost in 2021.

Dean Lee

Facility for Rare Isotope Beams and Department of Physics and Astronomy

Michigan State University, East Lansing, MI 48824, USA

E-mail: leed@frib.msu.edu 


\section{Early lattice calculations}

The idea of combining lattice simulations with effective field theory is quite natural. The lattice grid provides a short-distance regulator that can be used for an effective field theory. The earliest lattice calculations of nuclear systems were posed in momentum space [11. While this formulation of the lattice action makes the kinetic energy simple, it has the computational disadvantage that interaction terms require a summation over many momentum modes. The earliest lattice calculations using a spatial lattice studied the equation of state of nuclear matter with single-site and nearest-neighbor interactions 68 . Meanwhile, chiral perturbation theory for pions with lattice regularization was developed at around the same time [76, 60, and there were also lattice formulations of chiral symmetry involving static baryons [15].

The first lattice calculations involving dynamical nucleons with chiral effective field theory appeared in Ref. [55. In that paper, pions were also treated as dynamical fields that coupled to the nucleon fields. While pion-nucleon interactions produced the required pion-exchange interactions among the nucleons, they also altered the properties of individual nucleons such as the nucleon mass and the pion-nucleon coupling strength. These interactions of the dynamical pions were problematic since they were being iterated to all orders without their corresponding short-distance counterterms. Because of these complications, attention turned to simulations of pionless effective field theory, which didn't have such problems [56,57,58,7]. At the same time, several groups started applying lattice effective field theory to cold atoms and dilute neutron matter using pionless effective field theory [16, 12, 13, 85, 1]. This review, however, focuses on nuclear lattice simulations using chiral effective field theory.

Later it was realized that the problems with the pions could be cured by removing the time derivative of the pion field from the lattice action [4]. The resulting pion fields then only had spatial correlations without temporal correlations and produced the desired pion-exchange chiral potentials without modifying single nucleon properties and pion-nucleon vertices. This first study considered chiral effective field theory at leading order. The basic building block of the calculation is the Euclidean-time transfer matrix, $M . M$ is defined as the normal-ordered exponential of the lattice Hamiltonian $H$ over one time lattice step,

$$
M=: \exp \left[-H \alpha_{t}\right]:,
$$

where $\alpha_{t}$ is the ratio of the time lattice spacing, $a_{t}$, to the spatial lattice spacing, $a$. In the following, lattice units are used where all quantities are multiplied by powers of the spatial lattice spacing to make dimensionless combinations. One considers an initial state $\left|\Psi_{i}\right\rangle$ and final state $\left|\Psi_{f}\right\rangle$ that each have nonzero overlap with the nuclear state of interest. By multiplying by powers of $M$ upon $\left|\Psi_{i}\right\rangle$, the lowest energy state that couples to $\left|\Psi_{i}\right\rangle$ is enhanced. The projection amplitudes take the form

$$
Z_{f, i}\left(L_{t}\right)=\left\langle\Psi_{f}\left|M^{L_{t}}\right| \Psi_{i}\right\rangle .
$$


By calculating the ratio $Z_{f, i}\left(L_{t}\right) / Z_{f, i}\left(L_{t}-1\right)$ for large $L_{t}$, one can determine the energy of the lowest energy state. By using more than one initial and final state, a matrix of projection amplitudes can be used to compute excited states as well.

\section{Auxiliary fields and symmetries}

Auxiliary fields are used to generate the short-range nuclear interactions. The auxiliary field method can be viewed as a Gaussian integral formula connecting the exponential of the two-particle density, $\rho^{2}$, to the integral of the exponential of the one-particle density, $\rho$,

$$
: \exp \left(-\frac{c \alpha_{t}}{2} \rho^{2}\right):=\sqrt{\frac{1}{2 \pi}} \int_{-\infty}^{\infty} d s: \exp \left(-\frac{1}{2} s^{2}+\sqrt{-c \alpha_{t}} s \rho\right): .
$$

The normal ordering symbols :: indicate placing all annihilation operators to the right and all creation operators to the left, along with the corresponding minus sign for each operator order interchange. The normal ordering ensures that the operator products of the creation and annihilation operators behave as classical anticommuting Grassmann variables. Auxiliary fields are introduced at every lattice site to produce the required two-particle interactions [39,79, 45. Auxiliary fields can also be used to produce higher-body interactions [17. 46. The pion fields are treated in a manner similar to the auxiliary fields, except that the pion fields also have nontrivial correlations across different spatial lattice sites.

The amplitude for an $A$-body system is the determinant of an $A \times A$ matrix of single-nucleon amplitudes. The resulting lattice calculations consist of computing matrix determinants for all possible values of the auxiliary fields. These calculations are done using Markov Chain Monte Carlo sampling, and it is important that the determinant is not fluctuating strongly in sign or complex phase. Fortunately, the fact that the two S-wave nucleon-nucleon channels have approximately equal strength gives rise to Wigner's approximate SU(4) symmetry [84] of the low-energy nuclear interactions. This approximate SU(4) symmetry for the attractive S-wave interactions suppresses the sign and phase oscillations of the matrix determinants. This has been studied in several papers, some of which also consider inequalities that can be proven as a consequence [51,52, 17, 53, 38.

The direct simulations of the higher-order terms in chiral effective field theory cause problems with sign and phase oscillations. Some efforts were made to mitigate this problem using extrapolation methods [4], and others have taking a variational approach [86]. But most of the efforts avoid this problem by computing high-order terms using perturbation theory [6, 29, 30, 32, 31, 48, 62. In order to determine all the unknown short-range operator coefficients, it is necessary to compute nucleon-nucleon phase shifts on the lattice. While this can be done using Lüscher's finite volume method [67, the spherical wall method was found to provide greater accuracy for nucleon-nucleon scattering, 
especially for coupled partial-wave channels [5, 64, 10]. In addition to higherorder corrections in chiral effective field theory, corrections needed to restore Galilean invariance have also been introduced [61.

Numerous calculations of nuclear structure and many-body systems have been performed, in particular the structure of alpha cluster states such as the Hoyle state in ${ }^{12} \mathrm{C}$ [33, 26, 27, 75] as well as states in ${ }^{16} \mathrm{O}$ 28. As most other $a b$ initio methods have difficulty computing such highly-correlated alpha cluster states, these works provide strong scientific justification for continuing efforts in lattice chiral effective field theory. One important aspect of such calculations is the removal lattice artifacts from the final results. Towards this end, there have been several developments to restore rotational symmetry 63, 78, as well as remove finite-volume corrections from bound state calculations [8, 43, 18, 47 , 44 .

\section{Adiabatic projection method}

In order to compute nuclear scattering and reaction observables, a method called the adiabatic projection method was developed [69,71,23] and used to perform $a b$ initio calculations of alpha-alpha scattering [24. The adiabatic projection method constructs a low-energy effective theory for clusters of particles that is exact in the limit of large Euclidean time. Consider a set of two cluster states labelled according to their spatial separation vector $\mathbf{R}$. The initial wave functions are wave packets which, for large $|\mathbf{R}|$, factorize into a product of two individual clusters,

$$
|\mathbf{R}\rangle=\sum_{\mathbf{r}}|\mathbf{r}+\mathbf{R}\rangle_{1} \otimes|\mathbf{r}\rangle_{2}
$$

The summation over $\mathbf{r}$ produces states with total momentum equal to zero. The initial cluster states are binned together according to radial distance and angular momentum. This produces radial position states with angular momentum quantum numbers labelled as $|R\rangle^{J, J_{z}}$.

The next step is to multiply by powers of the transfer matrix in order to form "dressed" cluster states that approximately span the set of low-energy cluster-cluster scattering states in the periodic box of the lattice system. After $n_{t}$ time steps, the dressed cluster states are

$$
|R\rangle_{n_{t}}^{J, J_{z}}=M^{n_{t}}|R\rangle^{J, J_{z}}
$$

These dressed cluster states are then used to compute matrix elements of the transfer matrix $M$,

$$
\left[M_{n_{t}}\right]_{R^{\prime}, R}^{J, J_{z}}={ }_{n_{t}}^{J, J_{z}}\left\langle R^{\prime}|M| R\right\rangle_{n_{t}}^{J, J_{z}}
$$

Since such states are not orthogonal, it is also necessary to compute the norm matrix

$$
\left[N_{n_{t}}\right]_{R^{\prime}, R}^{J, J_{z}}={ }_{n_{t}}^{J, J_{z}}\left\langle R^{\prime} \mid R\right\rangle_{n_{t}}^{J, J_{z}}
$$


The "radial adiabatic transfer matrix" is defined as the matrix product

$$
\left[M_{n_{t}}^{a}\right]_{R^{\prime}, R}^{J, J_{z}}=\left[N_{n_{t}}^{-\frac{1}{2}} M_{n_{t}} N_{n_{t}}^{-\frac{1}{2}}\right]_{R^{\prime}, R}^{J, J_{z}},
$$

and a spherical hard wall boundary of radius $R_{W}$ is imposed. For large $n_{t}$, the standing waves of the radial adiabatic transfer matrix can be used to determine the elastic phase shifts. By including additional channels, the radial adiabatic transfer matrix can be used to compute inelastic reactions as well as capture reactions 73 .

\section{Pinholes for structure and thermodynamics}

The amplitude for each auxiliary field configuration involves quantum states which are superpositions of many different center-of-mass positions. Therefore, information about density correlations relative to the center of mass is not straightforward to obtain. The pinhole algorithm solves this problem by computing a classical probability distribution for the positions of the nucleons 22. Let $\rho_{i, j}(\mathbf{n})$ be the density operator for nucleons with spin $i$ and isospin $j$ at lattice site $\mathbf{n}$,

$$
\rho_{i, j}(\mathbf{n})=a_{i, j}^{\dagger}(\mathbf{n}) a_{i, j}(\mathbf{n}) .
$$

The normal-ordered $A$-body density operator is defined as

$$
\rho_{i_{1}, j_{1}, \cdots i_{A}, j_{A}}\left(\mathbf{n}_{1}, \cdots \mathbf{n}_{A}\right)=: \rho_{i_{1}, j_{1}}\left(\mathbf{n}_{1}\right) \cdots \rho_{i_{A}, j_{A}}\left(\mathbf{n}_{A}\right): .
$$

The $A$-body density operator is inserted in the middle of the Euclidean-time lattice simulations. The amplitude will vanish unless there are $A$ nucleons that exactly match the spatial positions $\mathbf{n}_{1}, \cdots \mathbf{n}_{A}$ and spin-isospin indices $i_{1}, j_{1}, \cdots i_{A}, j_{A}$. The spatial positions and spin-isospin indices can be viewed as "pinholes" in an otherwise impenetrable wall, and the pinhole positions and indices are updated using Monte Carlo sampling. This is shown in Fig. 1 . The pinhole algorithm has been used to compute the density distributions of protons and neutrons in various nuclei [22,66].

In the area of nuclear thermodynamics, there have been recent lattice calculations of spin and density correlations of neutron matter at nonzero temperature 2. A modified version of the pinhole algorithm called the pinhole trace algorithm was also developed for first principles calculations of nuclear thermodynamics 65. In the pinhole trace algorithm, the trace over $A$-nucleon states is computed in position space,

$\operatorname{Tr} O=\frac{1}{A !} \sum_{i_{1}, j_{1}, \mathbf{n}_{1} \cdots i_{A}, j_{A}, \mathbf{n}_{A}}\left\langle 0\left|a_{i_{A}, j_{A}}\left(\mathbf{n}_{A}\right) \cdots a_{i_{1}, j_{1}}\left(\mathbf{n}_{1}\right) O a_{i_{1}, j_{1}}^{\dagger}\left(\mathbf{n}_{1}\right) \cdots a_{i_{A}, j_{A}}^{\dagger}\left(\mathbf{n}_{A}\right)\right| 0\right\rangle$.

The pinhole trace algorithm was used to predict the liquid-vapor critical point in symmetric nuclear matter and probe alpha cluster formation as a function of temperature and density [65]. 


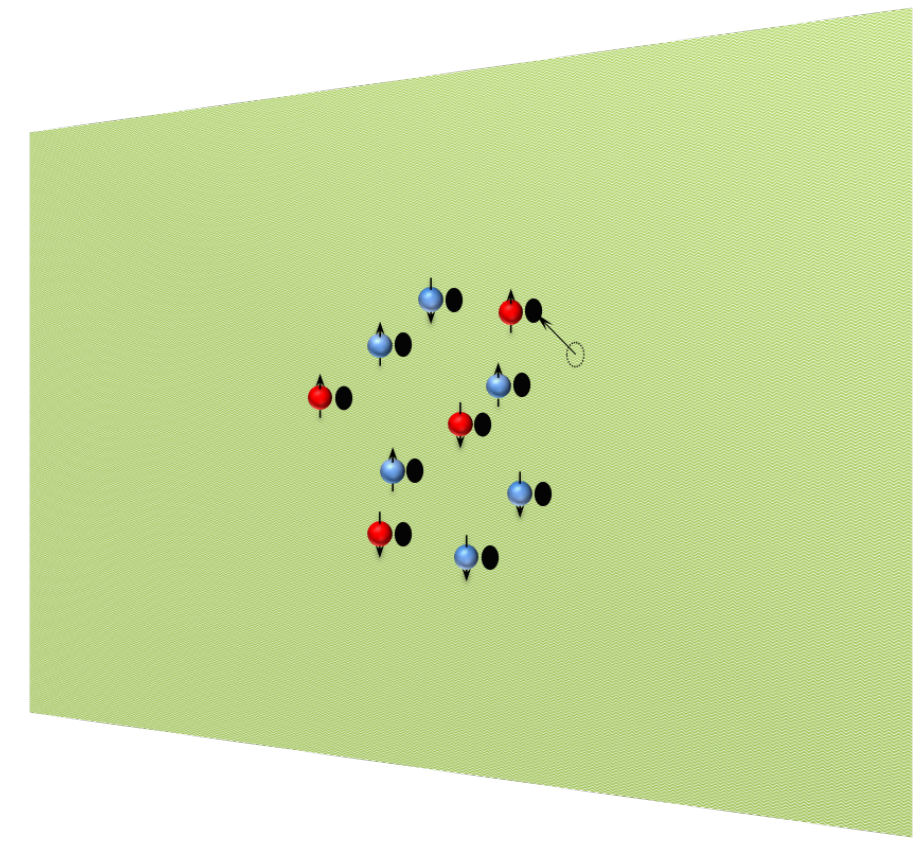

Fig. 1 Schematic drawing of nucleons and corresponding pinholes in the pinhole algorithm

\section{Nuclear forces and quantum phase transitions}

In addition to calculations using the most accurate lattice interactions available, there has also been a concerted effort to understand the most important characteristics of the nuclear force that determine the properties of nuclear binding. In Ref. [25], it was shown that nuclear physics resides near a quantum phase transition between a nuclear liquid and a Bose-Einstein condensate of alpha clusters. By changing the range and locality of the nuclear force, one can traverse from one phase to the other. The definition of locality here is that the nuclear interaction is diagonal with respect to particle position. In contrast, a nonlocal interaction is one that is off-diagonal with respect to nucleon positions. Fig. 2 shows the phase diagram of the ground state of symmetric nuclear matter as a function of a parameter $\lambda$ that controls the strength of the local part of the nuclear interactions. The vertical axis is the energy relative to the corresponding multi-alpha-particle threshold.

The mechanism for this dependence on range and locality arises from a competition between attractive nuclear forces and the Pauli repulsion between identical nucleons [70,40. The radius of the alpha particle sets a characteristic length scale where the Pauli repulsion between identical nucleons in symmetric nuclear matter becomes strong. The fact that nature appears to sit rather close to the quantum phase transition can be understood as arising from the phenomenological coincidence that the nucleon-nucleon interaction has an interaction range that is comparable to the radius of the alpha particle. These 


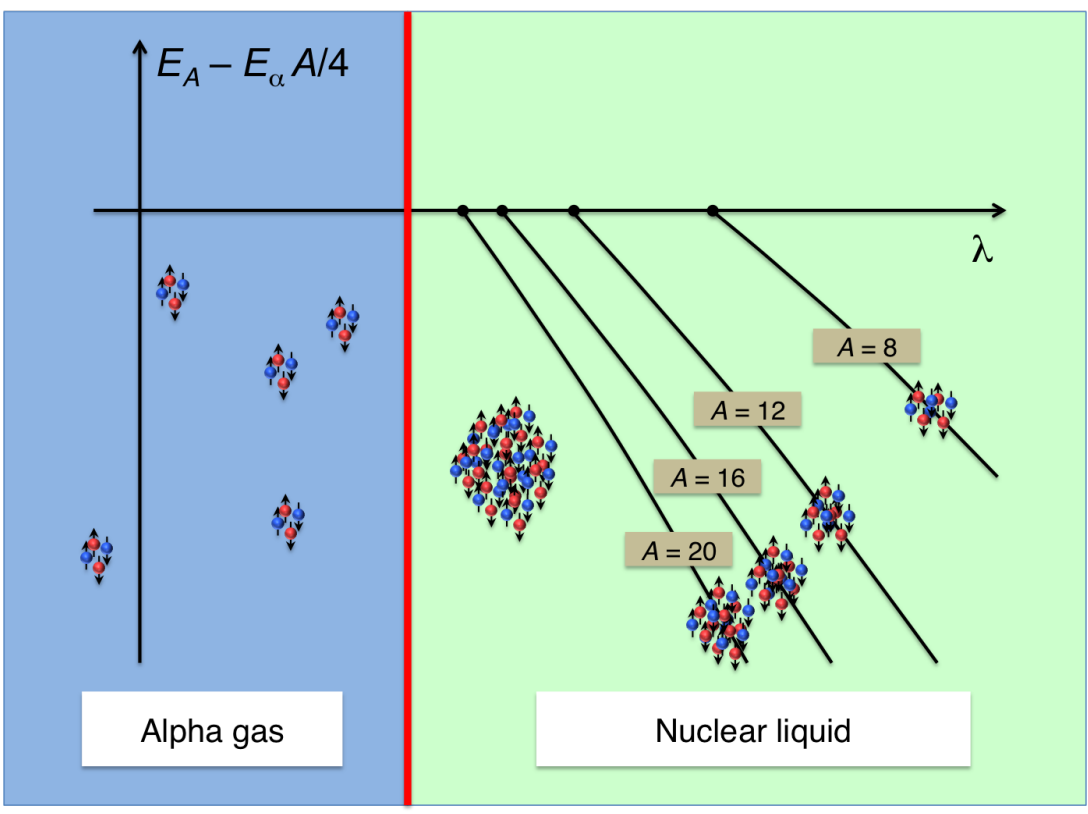

Fig. 2 Phase diagram of the ground state of symmetric nuclear matter as a function of the interpolating parameter $\lambda$ that controls the strength of the local part of the nuclear interactions.

findings were used to determine the simplest possible microscopic nuclear force that could accurately reproduce, on average, the ground state properties and charge radii of light and medium mass nuclei as well as neutron matter [66]. The interaction was surprisingly simple and, except for the Coulomb interaction, respected Wigner's SU(4) symmetry. This approximate SU(4) symmetry can be understood as resulting from an approximate spin-isospin exchange symmetry that appears when the number of colors in quantum chromodynamics is large [42, 41, 3, 14, 80, 72, 59. In Ref. [59, a derivation is given for the observations in Ref. [80,72 that there is an optimal resolution scale, corresponding to a momentum cutoff scale of about $500 \mathrm{MeV}$, where spin-isospin symmetry is manifest. These findings provide new insights into why chiral effective field theory performs remarkably well for momentum cutoff values near this scale. They also provide new insights into the success of nuclear lattice simulations, which rely on the approximate spin-isospin symmetry to keep sign and phase oscillations small.

\section{Summary and Emerging Directions}

Detailed reviews of lattice effective field theory can be found in Ref. [54,21, 50. This brief review gives an overview of progress in nuclear lattice simulations using chiral effective field theory. Nuclear lattice simulations is just one 
of many different methods to address the few-body and many-body problem in nuclear physics. Perhaps its most important feature is that its strengths and weaknesses are very different from those of other approaches and thus provides valuable new information. For example, nuclear lattice simulations is able to probe correlations in alpha cluster states and compute nuclear thermodynamics without much difficulty.

This review started with the early history of nuclear lattice simulations, and it discussed the auxiliary field formalism and the importance of symmetries in keeping sign and phase oscillations in check. The adiabatic projection method for nuclear scattering and reactions was reviewed, as well as the pinhole algorithm for structure and the pinhole trace algorithm for thermodynamics. It also presented a discussion of the connection between nuclear forces and nuclear binding and the proximity of a quantum phase transition.

There are many new directions in nuclear lattice simulations now under active development. The first lattice calculations using body-centered cubic lattices were recently completed [77. Lattice calculations were able to determine the condensate fraction of a superfluid in the unitary limit [37, and the first lattice calculations of hypernuclei were performed [35] using impurity lattice Monte Carlo [9. The combination of lattice simulations with eigenvector continuation holds promise for calculating higher-order without relying on perturbation theory $34,36,19,20,74$. All of these developments, as well as others still in progress, show that the field of nuclear lattice simulations using chiral effective field theory is progressing and growing in relevance.

Acknowledgements The author is grateful to Jose Manuel Alarcón, Buḡra Borasoy, Lukas Bovermann, Jiunn-Wei Chen, Dechuan Du, Joaquín Drut, Serdar Elhatisari, Evgeny Epelbaum, Dillon Frame, Rongzheng He, Youngman Kim, Nico Klein, Sebastian König, Hermann Krebs, Timo Lähde, Ning Li, Bing-Nan Lu, Yuanzhuo Ma, Ulf-G. Meißner, Michelle Pine, Alexander Rokash, Gautam Rupak, Avik Sarkar, Thomas Schäfer, Shihang Shen, Gianluca Stellin, Young-Ho Song, and all the members of the Nuclear Lattice Effective Field Theory Collaboration for their deep insights and productive collaboration. Special thanks also to Ulf-G. Meißner for his careful reading and comments on this review. Funding is gratefully acknowledged by the U.S. Department of Energy (DE-SC0013365 and DE-SC0021152) and the Nuclear Computational Low-Energy Initiative (NUCLEI) SciDAC-4 project (DESC0018083) as well as computational resources provided by the Oak Ridge Leadership Computing Facility through the INCITE award "Ab-initio nuclear structure and nuclear reactions", the Gauss Centre for Supercomputing e.V. (www.gauss-centre.eu) for computing time on the GCS Supercomputer JUWELS at Jülich Supercomputing Centre (JSC), the National Supercomputing Center at the Korea Institute for Science and Technology Information, and the high-performance computing centers at RWTH Aachen University and Michigan State University.

\section{References}

1. Abe, T., Seki, R.: Lattice calculation of thermal properties of low-density neutron matter with nn effective field theory. Phys. Rev. C79, 054,002 (2009)

2. Alexandru, A., Bedaque, P., Berkowitz, E., Warrington, N.C.: Structure Factors of Neutron Matter at Finite Temperature. Phys. Rev. Lett. 126(13), 132,701 (2021). DOI 10.1103/PhysRevLett.126.132701 
3. Banerjee, M.K., Cohen, T.D., Gelman, B.A.: The Nucleon nucleon interaction and large N(c) QCD. Phys. Rev. C 65, 034,011 (2002). DOI 10.1103/PhysRevC.65.034011

4. Borasoy, B., Epelbaum, E., Krebs, H., Lee, D., Meißner, U.G.: Lattice Simulations for Light Nuclei: Chiral Effective Field Theory at Leading Order. Eur. Phys. J. A 31, 105-123 (2007). DOI 10.1140/epja/i2006-10154-1

5. Borasoy, B., Epelbaum, E., Krebs, H., Lee, D., Meißner, U.G.: Two-particle scattering on the lattice: Phase shifts, spin-orbit coupling, and mixing angles. Eur. Phys. J. A 34, 185-196 (2007). DOI 10.1140/epja/i2007-10500-9

6. Borasoy, B., Epelbaum, E., Krebs, H., Lee, D., Meißner, U.G.: Dilute neutron matter on the lattice at next-to-leading order in chiral effective field theory. Eur. Phys. J. A 35, 357-367 (2008). DOI 10.1140/epja/i2008-10545-2

7. Borasoy, B., Krebs, H., Lee, D., Meißner, U.G.: The Triton and three-nucleon force in nuclear lattice simulations. Nucl. Phys. A 768, 179-193 (2006). DOI 10.1016/j. nuclphysa.2006.01.009

8. Bour, S., Koenig, S., Lee, D., Hammer, H.W., Meißner, U.G.: Topological phases for bound states moving in a finite volume. Phys. Rev. D 84, 091,503 (2011). DOI 10.1103/PhysRevD.84.091503

9. Bour, S., Lee, D., Hammer, H.W., Meißner, U.G.: Ab initio Lattice Results for Fermi Polarons in Two Dimensions. Phys. Rev. Lett. 115(18), 185,301 (2015). DOI 10.1103/ PhysRevLett.115.185301

10. Bovermann, L., Epelbaum, E., Krebs, H., Lee, D.: Scattering phase shifts and mixing angles for an arbitrary number of coupled channels on the lattice. Phys. Rev. C 100(6), 064,001 (2019). DOI 10.1103/PhysRevC.100.064001

11. Brockmann, R., Frank, J.: goo. Phys. Rev. Lett. 68, 1830-1833 (1992)

12. Bulgac, A., Drut, J.E., Magierski, P.: Spin 1/2 Fermions in the unitary regime: A Superfluid of a new type. Phys. Rev. Lett. 96, 090,404 (2006). DOI 10.1103/PhysRevLett. 96.090404

13. Burovski, E., Prokof'ev, N., Svistunov, B., Troyer, M.: The Fermi Hubbard model at unitarity. New Journal of Physics 8(8), 153 (2006). DOI 10.1088/1367-2630/8/8/153

14. Calle Cordon, A., Ruiz Arriola, E.: Serber symmetry, Large N(c) and Yukawa-like One Boson Exchange Potentials. Phys. Rev. C 80, 014,002 (2009). DOI 10.1103/PhysRevC. 80.014002

15. Chandrasekharan, S., Pepe, M., Steffen, F.D., Wiese, U.J.: Nonlinear realization of chiral symmetry on the lattice. JHEP 12, 035 (2003). DOI 10.1088/1126-6708/2003/12/035

16. Chen, J.W., Kaplan, D.B.: A Lattice theory for low-energy fermions at finite chemical potential. Phys. Rev. Lett. 92, 257,002 (2004). DOI 10.1103/PhysRevLett.92.257002

17. Chen, J.W., Lee, D., Schäfer, T.: Inequalities for light nuclei in the Wigner symmetry limit. Phys. Rev. Lett. 93, 242,302 (2004). DOI 10.1103/PhysRevLett.93.242302

18. Davoudi, Z., Savage, M.J.: Improving the Volume Dependence of Two-Body Binding Energies Calculated with Lattice QCD. Phys. Rev. D 84, 114,502 (2011). DOI 10.1103/ PhysRevD.84.114502

19. Demol, P., Duguet, T., Ekström, A., Frosini, M., Hebeler, K., König, S., Lee, D., Schwenk, A., Somà, V., Tichai, A.: Improved many-body expansions from eigenvector continuation. Phys. Rev. C 101(4), 041,302 (2020). DOI 10.1103/PhysRevC.101.041302

20. Demol, P., Frosini, M., Tichai, A., Somà, V., Duguet, T.: Bogoliubov many-body perturbation theory under constraint. Annals Phys. 424, 168,358 (2021). DOI 10.1016/j.aop.2020.168358

21. Drut, J.E., Nicholson, A.N.: Lattice methods for strongly interacting many-body systems. J. Phys. G: Nucl. Part. Phys. 40(4), 043101 (2013). DOI 10.1088/0954-3899/40/ 4/043101

22. Elhatisari, S., Epelbaum, E., Krebs, H., Lähde, T.A., Lee, D., Li, N., Lu, B.n., Meißner, U.G., Rupak, G.: Ab initio Calculations of the Isotopic Dependence of Nuclear Clustering. Phys. Rev. Lett. 119(22), 222,505 (2017). DOI 10.1103/PhysRevLett.119.222505

23. Elhatisari, S., Lee, D., Meißner, U.G., Rupak, G.: Nucleon-deuteron scattering using the adiabatic projection method. Eur. Phys. J. A 52(6), 174 (2016). DOI 10.1140/ epja/i2016-16174-2

24. Elhatisari, S., Lee, D., Rupak, G., Epelbaum, E., Krebs, H., Lähde, T.A., Luu, T., Meißner, U.G.: Ab initio alpha-alpha scattering. Nature 528, 111 (2015). DOI 10. $1038 /$ nature16067 
25. Elhatisari, S., et al.: Nuclear binding near a quantum phase transition. Phys. Rev. Lett. 117(13), 132,501 (2016). DOI 10.1103/PhysRevLett.117.132501

26. Epelbaum, E., Krebs, H., Lahde, T.A., Lee, D., Meißner, U.G.: Structure and rotations of the Hoyle state. Phys. Rev. Lett. 109, 252,501 (2012). DOI 10.1103/PhysRevLett. 109.252501

27. Epelbaum, E., Krebs, H., Lähde, T.A., Lee, D., Meißner, U.G.: Viability of CarbonBased Life as a Function of the Light Quark Mass. Phys. Rev. Lett. 110(11), 112,502 (2013). DOI 10.1103/PhysRevLett.110.112502

28. Epelbaum, E., Krebs, H., Lähde, T.A., Lee, D., Meißner, U.G., Rupak, G.: Ab Initio Calculation of the Spectrum and Structure of ${ }^{16}$ O. Phys. Rev. Lett. 112(10), 102,501 (2014). DOI 10.1103/PhysRevLett.112.102501

29. Epelbaum, E., Krebs, H., Lee, D., Meißner, U.G.: Ground state energy of dilute neutron matter at next-to-leading order in lattice chiral effective field theory. Eur. Phys. J. A 40, 199-213 (2009). DOI 10.1140/epja/i2009-10755-0

30. Epelbaum, E., Krebs, H., Lee, D., Meißner, U.G.: Lattice chiral effective field theory with three-body interactions at next-to-next-to-leading order. Eur. Phys. J. A 41, 125139 (2009). DOI 10.1140/epja/i2009-10764-y

31. Epelbaum, E., Krebs, H., Lee, D., Meißner, U.G.: Lattice calculations for A=3,4,6,12 nuclei using chiral effective field theory. Eur. Phys. J. A 45, 335-352 (2010). DOI 10.1140/epja/i2010-11009-x

32. Epelbaum, E., Krebs, H., Lee, D., Meißner, U.G.: Lattice effective field theory calculations for $\mathrm{A}=3,4,6,12$ nuclei. Phys. Rev. Lett. 104, 142,501 (2010). DOI 10.1103/PhysRevLett.104.142501

33. Epelbaum, E., Krebs, H., Lee, D., Meißner, U.G.: Ab initio calculation of the Hoyle state. Phys. Rev. Lett. 106, 192,501 (2011). DOI 10.1103/PhysRevLett.106.192501

34. Frame, D., He, R., Ipsen, I., Lee, D., Lee, D., Rrapaj, E.: Eigenvector continuation with subspace learning. Phys. Rev. Lett. 121(3), 032,501 (2018). DOI 10.1103/PhysRevLett. 121.032501

35. Frame, D., Lähde, T.A., Lee, D., Meißner, U.G.: Impurity Lattice Monte Carlo for Hypernuclei. Eur. Phys. J. A 56(10), 248 (2020). DOI 10.1140/epja/s10050-020-00257-y

36. Frame, D.K.: Ab Initio Simulations of Light Nuclear Systems Using Eigenvector Continuation and Auxiliary Field Monte Carlo. Other thesis (2019)

37. He, R., Li, N., Lu, B.N., Lee, D.: Superfluid Condensate Fraction and Pairing Wave Function of the Unitary Fermi Gas. Phys. Rev. A 101(6), 063,615 (2020). DOI 10.1103/PhysRevA.101.063615

38. Hoffman, M.D., Loheac, A.C., Porter, W.J., Drut, J.E.: Thermodynamics of onedimensional SU(4) and SU(6) fermions with attractive interactions. Phys. Rev. A 95(3), 033,602 (2017). DOI 10.1103/PhysRevA.95.033602

39. Hubbard, J.: Calculation of partition functions. Phys. Rev. Lett. 3, 77-80 (1959). DOI 10.1103/PhysRevLett.3.77

40. Kanada-En'yo, Y., Lee, D.: Effective interactions between nuclear clusters. Phys. Rev. C 103(2), 024,318 (2021). DOI 10.1103/PhysRevC.103.024318

41. Kaplan, D.B., Manohar, A.V.: The Nucleon-nucleon potential in the $1 / \mathrm{N}(\mathrm{c})$ expansion. Phys. Rev. C 56, 76-83 (1997). DOI 10.1103/PhysRevC.56.76

42. Kaplan, D.B., Savage, M.J.: The Spin flavor dependence of nuclear forces from large $n$ QCD. Phys. Lett. B 365, 244-251 (1996). DOI 10.1016/0370-2693(95)01277-X

43. Koenig, S., Lee, D., Hammer, H.W.: Non-relativistic bound states in a finite volume. Annals Phys. 327, 1450-1471 (2012). DOI 10.1016/j.aop.2011.12.015

44. König, S., Lee, D.: Volume Dependence of N-Body Bound States. Phys. Lett. B 779, 9-15 (2018). DOI 10.1016/j.physletb.2018.01.060

45. Koonin, S.E.: Auxiliary-field Monte Carlo methods. Journal of Statistical Physics 43(56), 985-990 (1986). DOI 10.1007/BF02628325

46. Körber, C., Berkowitz, E., Luu, T.: Sampling General N-Body Interactions with Auxiliary Fields. EPL 119(6), 60,006 (2017). DOI 10.1209/0295-5075/119/60006

47. Körber, C., Luu, T.: Applying Twisted Boundary Conditions for Few-body Nuclear Systems. Phys. Rev. C 93(5), 054,002 (2016). DOI 10.1103/PhysRevC.93.054002

48. Lähde, T.A., Epelbaum, E., Krebs, H., Lee, D., Meißner, U.G., Rupak, G.: Lattice Effective Field Theory for Medium-Mass Nuclei. Phys. Lett. B 732, 110-115 (2014). DOI 10.1016/j.physletb.2014.03.023 
49. Lähde, T.A., Luu, T., Lee, D., Meißner, U.G., Epelbaum, E., Krebs, H., Rupak, G.: Nuclear Lattice Simulations using Symmetry-Sign Extrapolation. Eur. Phys. J. A 51(7), 92 (2015). DOI 10.1140/epja/i2015-15092-1

50. Lähde, T.A., Meißner, U.G.: Nuclear Lattice Effective Field Theory: An introduction, vol. 957. Springer (2019). DOI 10.1007/978-3-030-14189-9

51. Lee, D.: Inequalities for low-energy symmetric nuclear matter. Phys. Rev. C 70, 064,002 (2004). DOI 10.1103/PhysRevC.70.064002

52. Lee, D.: Pressure inequalities for nuclear and neutron matter. Phys. Rev. C 71, 044,001 (2005). DOI 10.1103/PhysRevC.71.044001

53. Lee, D.: Spectral convexity for attractive SU(2N) fermions. Phys. Rev. Lett. 98, 182,501 (2007). DOI 10.1103/PhysRevLett.98.182501

54. Lee, D.: Lattice simulations for few- and many-body systems. Prog. Part. Nucl. Phys. 63, 117-154 (2009). DOI 10.1016/j.ppnp.2008.12.001

55. Lee, D., Borasoy, B., Schäfer, T.: Nuclear lattice simulations with chiral effective field theory. Phys. Rev. C 70, 014,007 (2004). DOI 10.1103/PhysRevC.70.014007

56. Lee, D., Schäfer, T.: Neutron matter on the lattice with pionless effective field theory. Phys. Rev. C 72, 024,006 (2005). DOI 10.1103/PhysRevC.72.024006

57. Lee, D., Schäfer, T.: Cold dilute neutron matter on the lattice. I. Lattice virial coefficients and large scattering lengths. Phys. Rev. C 73, 015,201 (2006). DOI 10.1103/PhysRevC.73.015201

58. Lee, D., Schäfer, T.: Cold dilute neutron matter on the lattice. II. Results in the unitary limit. Phys. Rev. C 73, 015,202 (2006). DOI 10.1103/PhysRevC.73.015202

59. Lee, D., et al.: Hidden Spin-Isospin Exchange Symmetry. Phys. Rev. Lett. 127(6), 062,501 (2021). DOI 10.1103/PhysRevLett.127.062501

60. Lewis, R., Ouimet, P.P.A.: Lattice regularization for chiral perturbation theory. Phys. Rev. D 64, 034,005 (2001). DOI 10.1103/PhysRevD.64.034005

61. Li, N., Elhatisari, S., Epelbaum, E., Lee, D., Lu, B., Meißner, U.G.: Galilean invariance restoration on the lattice. Phys. Rev. C 99(6), 064,001 (2019). DOI 10.1103/PhysRevC. 99.064001

62. Li, N., Elhatisari, S., Epelbaum, E., Lee, D., Lu, B.N., Meißner, U.G.: Neutron-proton scattering with lattice chiral effective field theory at next-to-next-to-next-to-leading order. Phys. Rev. C 98(4), 044,002 (2018). DOI 10.1103/PhysRevC.98.044002

63. Lu, B.N., Lähde, T.A., Lee, D., Meißner, U.G.: Breaking and restoration of rotational symmetry on the lattice for bound state multiplets. Phys. Rev. D 90(3), 034,507 (2014). DOI 10.1103/PhysRevD.90.034507

64. Lu, B.N., Lähde, T.A., Lee, D., Meißner, U.G.: Precise determination of lattice phase shifts and mixing angles. Phys. Lett. B 760, 309-313 (2016). DOI 10.1016/j.physletb. 2016.06.081

65. Lu, B.N., Li, N., Elhatisari, S., Lee, D., Drut, J.E., Lähde, T.A., Epelbaum, E., Meißner, U.G.: AbInitio Nuclear Thermodynamics. Phys. Rev. Lett. 125(19), 192,502 (2020). DOI 10.1103/PhysRevLett.125.192502

66. Lu, B.N., Li, N., Elhatisari, S., Lee, D., Epelbaum, E., Meißner, U.G.: Essential elements for nuclear binding. Phys. Lett. B 797, 134,863 (2019). DOI 10.1016/j.physletb.2019. 134863

67. Luscher, M.: Volume Dependence of the Energy Spectrum in Massive Quantum Field Theories. 2. Scattering States. Commun. Math. Phys. 105, 153-188 (1986). DOI 10.1007/BF01211097

68. Muller, H.M., Koonin, S.E., Seki, R., van Kolck, U.: Nuclear matter on a lattice. Phys. Rev. C 61, 044,320 (2000). DOI 10.1103/PhysRevC.61.044320

69. Pine, M., Lee, D., Rupak, G.: Adiabatic projection method for scattering and reactions on the lattice. Eur. Phys. J. A 49, 151 (2013). DOI 10.1140/epja/i2013-13151-3

70. Rokash, A., Epelbaum, E., Krebs, H., Lee, D.: Effective forces between quantum bound states. Phys. Rev. Lett. 118(23), 232,502 (2017). DOI 10.1103/PhysRevLett.118.232502

71. Rokash, A., Pine, M., Elhatisari, S., Lee, D., Epelbaum, E., Krebs, H.: Scattering cluster wave functions on the lattice using the adiabatic projection method. Phys. Rev. C 92(5), 054,612 (2015). DOI 10.1103/PhysRevC.92.054612

72. Ruiz Arriola, E.: Low Scale Saturation of Effective NN Interactions and Their Symmetries. Symmetry 8(6), 42 (2016). DOI 10.3390/sym8060042 
73. Rupak, G., Lee, D.: Radiative capture reactions in lattice effective field theory. Phys. Rev. Lett. 111(3), 032,502 (2013). DOI 10.1103/PhysRevLett.111.032502

74. Sarkar, A., Lee, D.: Convergence of Eigenvector Continuation. Phys. Rev. Lett. 126(3), 032,501 (2021). DOI 10.1103/PhysRevLett.126.032501

75. Shen, S., Lähde, T.A., Lee, D., Meißner, U.G.: Wigner SU(4) symmetry, clustering, and the spectrum of ${ }^{12} \mathrm{C}(2021)$

76. Shushpanov, I.A., Smilga, A.V.: Chiral perturbation theory with lattice regularization. Phys. Rev. D 59, 054,013 (1999). DOI 10.1103/PhysRevD.59.054013

77. Song, Y.H., Kim, Y., Li, N., Lu, B.N., He, R., Lee, D.: Quantum Many-Body Calculations using Body-Centered Cubic Lattices (2021)

78. Stellin, G., Elhatisari, S., Meißner, U.G.: Breaking and restoration of rotational symmetry in the low-energy spectrum of light alpha-conjugate nuclei on the lattice I: ${ }^{8} \mathrm{Be}$ and ${ }^{12}$ C. Eur. Phys. J. A 54(12), 232 (2018). DOI 10.1140/epja/i2018-12671-6

79. Stratonovich, R.L.: On a method of calculating quantum distribution functions. Soviet Phys. Doklady 2, 416-419 (1958)

80. Timoteo, V., Szpigel, S., Ruiz Arriola, E.: Symmetries of the Similarity Renormalization Group for Nuclear Forces. Phys. Rev. C 86, 034,002 (2012). DOI 10.1103/PhysRevC. 86.034002

81. Weinberg, S.: Nuclear forces from chiral lagrangians. Phys. Lett. B251, 288-292 (1990)

82. Weinberg, S.: Effective chiral lagrangians for nucleon - pion interactions and nuclear forces. Nucl. Phys. B363, 3-18 (1991)

83. Weinberg, S.: Three body interactions among nucleons and pions. Phys. Lett. B295 114-121 (1992)

84. Wigner, E.: On the Consequences of the Symmetry of the Nuclear Hamiltonian on the Spectroscopy of Nuclei. Phys. Rev. 51, 106-119 (1937). DOI 10.1103/PhysRev.51.106

85. Wingate, M.: Field theoretic study of a cold Fermi gas in the unitary limit. PoS LAT2006, 153 (2006). DOI 10.22323/1.032.0153

86. Wlazłowski, G., Holt, J.W., Moroz, S., Bulgac, A., Roche, K.J.: Auxiliary-Field Quantum Monte Carlo Simulations of Neutron Matter in Chiral Effective Field Theory. Phys. Rev. Lett. 113(18), 182,503 (2014). DOI 10.1103/PhysRevLett.113.182503 